\title{
Slow-Goldstone mode generated by order from quantum disorder and its experimental detection
}

\author{
Fadi Sun ${ }^{a, b}$ and Jinwu Ye $\mathbf{Y}^{a, b, c}$ \\ ${ }^{a}$ Tsung-Dao Lee Institute, \\ Shanghai 200240, China \\ ${ }^{b}$ Institute for Quantum Science and Engineering, \\ Shenzhen 518055, China \\ ${ }^{c}$ Department of Physics and Astronomy, Mississippi State University, \\ Mississippi State, MS 39762, U.S.A. \\ E-mail: fs306@msstate.edu, jy306@msstate.edu
}

ABSTRACT: The order from quantum disorders (OFQD) phenomenon is well-known and ubiquitous in particle physics and frustrated magnetic systems. Typically, OFQD transfers a spurious Goldstone mode into a pseudo-Goldstone mode with a tiny gap. Here, we report an opposite phenomenon: OFQD transfers a spurious quadratic mode into a true linear Goldstone mode with a very small velocity (named slow-Goldstone mode). This new phenomenon is demonstrated in an interacting bosonic system subjected to an Abelian flux. We develop a new and systematic OFQD analysis to determine the true quantum ground state and the whole excitation spectrum. In the weak-coupling limit, the superfluid ground state has a 4 -sublattice $90^{\circ}$ coplanar spin structure, which supports 4 linear Goldstone modes with 3 different velocities. One of which is generated by the OFQD is much softer than the other 3 Goldstone modes, so it can be easily detected in the cold atom or photonic experiments. In the strong-coupling limit, the ferromagnetic Mott ground state with a true quadratic Goldstone mode. We speculate that there could be some topological phases intervening between the two symmetry broken states. These novel phenomena may be observed in the current cold-atom or photonic experiments subjected to an Abelian flux at the weak coupling limit where the heatings may be well under control. Possible connections to Coleman-Weinberg potential in particle physics, $1 / N$ expansion of Sachdev-Ye-Kitaev models and zero temperature quantum black hole entropy are outlined.

Keywords: Effective Field Theories, Field Theories in Lower Dimensions, Lattice Quantum Field Theory, Nonperturbative Effects

ArXiv EPrint: 1711.06304 


\section{Contents}

$\begin{array}{llr}1 & \text { Introduction } & 1\end{array}$

2 The model and two-steps order from quantum disorder analysis $\quad 3$

2.1 Order from quantum disorder mechanism to determine the quantum ground state: step 1

2.2 Excitation spectrum above the $90^{\circ}$ frustrated quantum ground state: a partial contradiction to the Goldstone theorem

2.3 The slow-Goldstone mode generated by the OFQD mechanism: step 2, a complete path integral formalism of the OFQD

$\begin{array}{ll}2.4 & \text { The condensate fraction and the quantum depletions } \\ \end{array}$

$\begin{array}{ll}2.5 & \text { The strong coupling ferromagnetic Mott state } \\ \end{array}$

3 The implications on cold atom and photonic experiments 11

4 Discussion and conclusion $\quad 12$

A The second step of the newly developed systematic "order from quantum disorder analysis" to compute the spectra of the slow-Goldstone mode: canonical quantization approach

B The kinetic energies and currents in the 4-sublattice $90^{\circ}$ coplanar ground state

\section{Introduction}

The Goldstone theorem states [1-3] that if a system has a continuous global symmetry $G$ which is spontaneously broken by its ground state to a smaller symmetry group $H$, then 1 ) the symmetry broken ground state must support gapless modes, 2) the number of which is just the difference of the number of generators of $G$ and $H$. These gapless modes with linear dispersions are called Goldstone modes. The Goldstone theorem has had tremendous applications in essentially all the branches of physics such as string theory/quantum gravity $[4,5]$, especially the gapless reparametrization mode in the Sachdev-Ye-Kitaev (SYK) models leading to its maximal chaos tying that of a quantum black hole [6-9], particle physics [10], condensed matter systems [11, 12], cold-atoms/quantum optics [13-15] and quantum information science [16]. The number of Goldstone modes can be counted just from the symmetry breaking analysis. However, it remains a challenge to evaluate their velocities on any specific Hamiltonian. 
On the other hand, the order from quantum disorder phenomena (OFQD) is quite common in particle physics [17-19] and frustrated magnetic systems [20-26]. It says that there are infinitely many degenerate ground states at the classical level due to a spurious continuous symmetry. There may also be an associated spurious Goldstone mode resulting from the breaking of such a spurious continuous symmetry. However, quantum fluctuations can pick up quantum ground states from this classically degenerate manifold and generate a small gap to the spurious Goldstone mode, therefore transfer it into a pseudo-Goldstone mode. Here we discover a completely opposite new phenomenon: the OFQD generates a true Goldstone mode with a very small velocity (named slow-Goldstone mode) which can be observed in the current spinor cold atoms [27-36] or photonic experiments [37-40] subjected to an Abelian $\alpha$ flux in the weak coupling regime.

The Abelian flux provides a frustrating source even in a square lattice which is entirely different from the geometric frustrations which only happen in frustrated lattices. For simplicity and practical relevance to the cold atom or photon experiments, we focus on the most frustrated case with $\alpha=\pi$ flux. We find that in the weak interaction limit, the $\pi$ flux sparks dramatic interplay between the superfluid (SF) and the (pseudo-)spin of spinor bosons, which leads to a frustrated superfluid even in a bipartite lattice. We develop a new and systematic two-step "order from quantum disorder" (OFQD) analysis to determine not only the ground state, but also the whole excitation spectrum of such a frustrated superfluid. In the first step, by identifying a classically degenerate family of states upto the exact spin $\mathrm{SU}(2)_{s}$ symmetry, and generalizing Bogoliubov theory to multi-components on a lattice, we find the quantum ground state is a superfluid state with the spin-orbital structure of a 4 -sublattice $90^{\circ}$ coplanar state in a square lattice. It not only breaks the charge $\mathrm{U}(1)_{c}$ symmetry, but also the spin $\mathrm{SU}(2)_{s}$ symmetry completely. The Goldstone theorem dictates that there should be 4 linear gapless modes. However, we find only 3 linear gapless modes, plus 1 quadratic mode in the long wavelength limit, in contradiction with the Goldstone theorem. Then we devise the second step to incorporate the effective potential generated by the OFQD into the Hamiltonian. It transfers the quadratic mode into a soft linear gapless mode which is the Goldstone mode generated by the OFQD, but the other three linear modes remain intact, in agreement with the exact results from the Goldstone theorem. Because the Goldstone mode generated by OFQD is much softer than the other three ones in the weak coupling, so it can be easily identified in the spinor cold atom or photonic experiments by various currently available detection methods. At the leading order of the strong coupling expansion, we find a ferromagnetic (FM) Mott state, now with a true quadratic FM mode. Due to the dramatically different nature of the two states in the weak and strong coupling, we speculate there could be a topological quantum spin liquid intervening between the weak coupling frustrated SF and the strong coupling FM Mott state. Because the heating effects can be easily suppressed at weak couplings, so this novel phenomenon due to OFQD at weak couplings can be easily observed in recent cold atom (or photonic) experiments generating Abelian flux in an optical lattice (or in a microwave cavity array). Several perspectives on Coleman-Weinberg potential in particle physics, $1 / N$ expansion of Sachdev-Ye-Kitaev, and the quantum fluctuations effects at zero temperature on Bekenstein-Hawking entropy are outlined. 


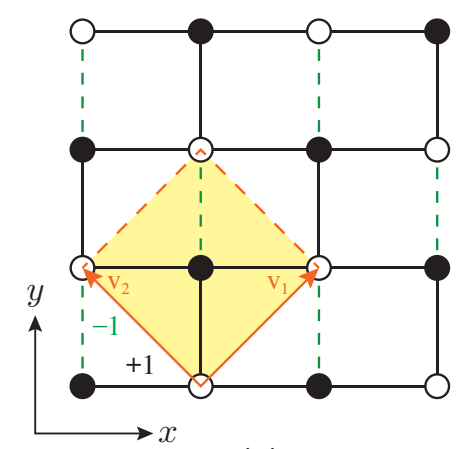

(a)

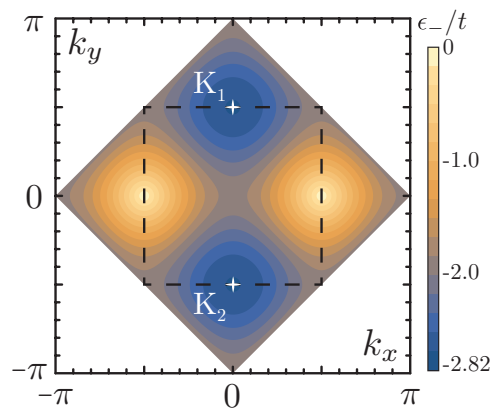

(b)

Figure 1. (a) The $\mathrm{Z}_{2}$ gauge used in the main text with the real and spin-independent hopping $-t e^{i A_{i j}}= \pm t$, which leads to two sites per unit cell. The frustrated bond with $e^{i A_{i j}}=-1$ is denoted by a dashed line. (b) In the $\mathrm{Z}_{2}$ gauge, there are two (doubly) degenerate minima at the two momentum $\mathbf{K}_{1}=(0, \pi / 2)$ and $\mathbf{K}_{2}=(0,-\pi / 2)$. The diamond shape is the Brillouin zone (BZ). The inner dashed line is $1 / 2$ of the BZ. The full square is twice the BZ (2BZ).

\section{The model and two-steps order from quantum disorder analysis}

We consider a pseudo-spin-1/2 Boson-Hubbard model in a $\pi$-flux on the square lattice described by:

$$
\mathcal{H}=-t \sum_{\langle i j\rangle, \sigma}\left(e^{i A_{i j}} b_{i \sigma}^{\dagger} b_{j \sigma}+\text { h.c. }\right)+\frac{U}{2} \sum_{i} n_{i}^{2}-\mu \sum_{i} n_{i}
$$

where $\langle i j\rangle$ indicates the nearest-neighbor sites of a square lattice, $b_{i \sigma}\left(b_{i \sigma}^{\dagger}\right)$ is the boson annihilation (creation) operators at site $i$ with a spin $\sigma, A_{i j}$ is the static gauge potential on the link [see figure 1(a)], $n_{i}=n_{i \uparrow}+n_{i \downarrow}$ is the total number of bosons for site $i$, and $\mu$ is a chemical potential. The second term with $U>0$ represents a repulsive on-site BoseHubbard interaction, which is invariant under spin $\mathrm{SU}(2)$ symmetry. As a result, the total Hamiltonian enjoys $\mathrm{U}(1)_{c} \times \mathrm{SU}(2)_{s}$ symmetry.

\subsection{Order from quantum disorder mechanism to determine the quantum ground state: step 1}

In the weak coupling limit $U / t \ll 1$, we will first diagonalize the non-interacting Hamiltonian and then treat the weak interaction perturbatively by generalizing Bogoliubov theory to two components. The gauge is shown in figure 1(a) leads to two sublattices $A$ and $B$. The diagonalization in this gauge leads to single-particle spectrum $\epsilon_{ \pm, \sigma}(\mathbf{k})=$ $\pm 2 t \sqrt{\cos ^{2} k_{x}+\sin ^{2} k_{y}}$, which develops two energy minima at momentum $\mathbf{K}_{1}=(0, \pi / 2)$ and $\mathbf{K}_{2}=(0,-\pi / 2)$ shown in figure $1(\mathrm{~b})$ with the corresponding two-sublattice eigenvectors:

$$
\eta_{1}=\frac{1}{\sqrt{2}}\left(\begin{array}{c}
e^{-i \frac{\pi}{4}} \\
1
\end{array}\right), \quad \eta_{2}=\frac{1}{\sqrt{2}}\left(\begin{array}{c}
e^{i \frac{\pi}{4}} \\
1
\end{array}\right) .
$$

At the weak coupling, the ground-state is a superfluid state, and all bosons will condense into the lowest single-particle energy eigenstate. In the presence of two degenerated 


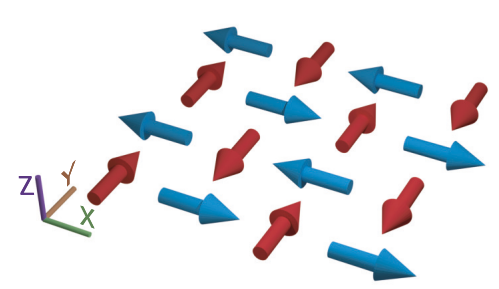

(a)

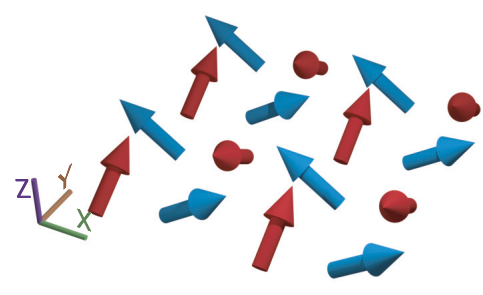

(b)

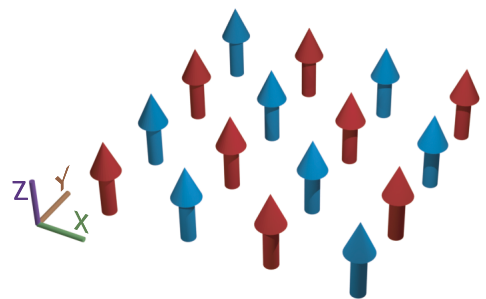

(c)

Figure 2. The spin structure of the superfluid (SF) states in the classically $\mathrm{U}(1)$ degenerate manifold at several representative $\phi$. (a) $\phi=\pi / 4$ (coplanar), (b) $\phi=\pi / 10$ (non-coplanar), (c) $\phi=0$ (collinear) ferromagnetism (FM), respectively. The order from the quantum disorder (OFQD) effect selects $\phi=\pi / 4$ as the true quantum ground state, which has the 4 -sublattice coplanar $90^{\circ}$ frustrated spin structure given by eq. (2.14). If changing the sign of the OFQD term, then it becomes the FM SF state (See ref. [41]).

two degenerate energy minima with momentum $\mathbf{K}_{1}=-\mathbf{K}_{2}=\mathbf{K}$, we can construct a condensate wave-function as

$$
\Psi_{\mathbf{r}}=\sqrt{2 n} e^{i \chi}\left[\left(\eta_{1} \otimes \mathbf{z}_{1}\right) e^{-i \mathbf{K} \cdot \mathbf{r}}+\left(\eta_{2} \otimes \mathbf{z}_{2}\right) e^{+i \mathbf{K} \cdot \mathbf{r}}\right]
$$

where $\Psi_{\mathbf{r}}=\left(b_{\mathbf{r}, A, \uparrow}, b_{\mathbf{r}, A, \downarrow}, b_{\mathbf{r}, B, \uparrow}, b_{\mathbf{r}, B, \downarrow}\right)^{\top}$ is a four-component spinor, $n$ is condensate density, $\eta_{1,2}$ are sublattice eigen-vectors defined in eq. (2.2), and $\mathbf{z}_{i}=\left(z_{i \uparrow}, z_{i \downarrow}\right)^{\top}$ with $i=1,2$ are two-component non-normalized spinors satisfying $\left|\mathbf{z}_{1}\right|^{2}+\left|\mathbf{z}_{2}\right|^{2}=1$. From the construction, $\Psi_{\mathbf{r}}$ with arbitrary $\mathbf{z}_{1,2}$ automatically minimizes Kinetic energy, but it gives different BoseHubbard interaction energy density

$$
E_{\text {int }}=\frac{n^{2} U}{2}\left(1+2\left|\mathbf{z}_{1}^{\dagger} \mathbf{z}_{2}\right|^{2}\right)
$$

which suggests the mean-field ground-state manifold is described by the condition $\mathbf{z}_{1}^{\dagger} \mathbf{z}_{2}=0$.

At the mean-field level, the condition $\mathbf{z}_{1}^{\dagger} \mathbf{z}_{2}=0$ leads to a degenerate family of groundstate, which contains not only the exact $\mathrm{U}(1)_{c} \times \mathrm{SU}(2)_{s}$ symmetries but also a spurious $\mathrm{U}(1)$ symmetry:

$$
\Psi_{0}=\sqrt{2 n_{0}} e^{i \chi}\left[\left(\eta_{1} \otimes \mathbf{z}_{1}^{(0)}\right) e^{-i \mathbf{K} \cdot \mathbf{r}}+\left(\eta_{2} \otimes \mathbf{z}_{2}^{(0)}\right) e^{+i \mathbf{K} \cdot \mathbf{r}}\right]
$$

where we fixed two spinors to be orthogonal

$$
\mathbf{z}_{1}^{(0)}=e^{-i \alpha} \cos \phi\left(\begin{array}{c}
e^{-i \gamma / 2} \cos \beta / 2 \\
e^{i \gamma / 2} \sin \beta / 2
\end{array}\right), \quad \mathbf{z}_{2}^{(0)}=e^{+i \alpha} \sin \phi\left(\begin{array}{c}
-e^{-i \gamma / 2} \sin \beta / 2 \\
e^{i \gamma / 2} \cos \beta / 2
\end{array}\right)
$$

The exact $\mathrm{U}(1)_{c}$ symmetry tunes the global phase $\chi$. The exact $\mathrm{SU}(2)_{s}$ symmetry tunes the three spin angles $(\alpha, \beta, \gamma)$, while the spurious $\mathrm{U}(1)$ symmetry tunes the angle $\phi$, which need to be determined by the "order from quantum disorder" mechanism. Three representative classically degenerate states are given in figure 2 . 
Using the exact $\mathrm{U}(1)_{c}$ symmetry and the exact $\mathrm{SU}(2)_{s}$ symmetry, we set $\chi=0$ and align the spinor along the spin- $Z$ direction. Equation (2.5) can be simplified to

$$
\Psi_{0}=\sqrt{\frac{N_{0}}{N_{s}}}\left[\cos \phi\left(\begin{array}{c}
e^{-i \frac{\pi}{4}} \\
1
\end{array}\right) \otimes\left(\begin{array}{l}
1 \\
0
\end{array}\right) e^{-i \mathbf{K} \cdot \mathbf{r}}+\sin \phi\left(\begin{array}{c}
e^{i \frac{\pi}{4}} \\
1
\end{array}\right) \otimes\left(\begin{array}{l}
0 \\
1
\end{array}\right) e^{+i \mathbf{K} \cdot \mathbf{r}}\right],
$$

where $N_{s}$ stand for the total number of lattice sites, and $N_{0}$ stand for the total number of condensed atoms, and $n_{0}=N_{0} / N_{s}$ is the condensate density. The density and spin of the state eq. (2.7) can be evaluated as:

$$
\begin{aligned}
& \left\langle\Psi_{0}^{s}\left|\sigma_{0}\right| \Psi_{0}^{s}\right\rangle=n_{0} ; \\
& \left\langle\Psi_{0}^{s}\left|\sigma_{x}\right| \Psi_{0}^{s}\right\rangle=n_{0} \cos (2 \mathbf{K} \cdot \mathbf{r}) \sin 2 \phi, 0 \quad s \in B, A ; \\
& \left\langle\Psi_{0}^{s}\left|\sigma_{y}\right| \Psi_{0}^{s}\right\rangle=n_{0} \cos (2 \mathbf{K} \cdot \mathbf{r}) \sin 2 \phi, 0 \quad s \in A, B ; \\
& \left\langle\Psi_{0}^{s}\left|\sigma_{z}\right| \Psi_{0}^{s}\right\rangle=n_{0} \cos 2 \phi .
\end{aligned}
$$

where $\mathbf{r}$ stands for the unit cell coordinate in figure 1(a) and $s=A, B$ stands for the two sublattices, i.e. $\mathbf{r}_{B}=\mathbf{r}_{A}+\hat{y}$. In terms of the coordinate- $i$ in the square lattice, the state has the spin structure $\mathbf{S}_{i}=\left(S_{i}^{x}, S_{i}^{y}, S_{i}^{z}\right)$ :

$$
\mathbf{S}_{i}=\frac{n_{0}}{2}\left(\frac{(-1)^{i_{x}}-(-1)^{i_{y}}}{2} \sin 2 \phi, \frac{(-1)^{i_{x}}+(-1)^{i_{y}}}{2} \sin 2 \phi, \cos 2 \phi\right) .
$$

Setting $\phi=0, \pi / 10, \pi / 4$ lead to the three typical states in figure 2

By writing the Bose field as a sum of condensation plus a small quantum fluctuation $\Psi=\Psi_{0}+\psi$, one can perform an expansion of original Hamiltonian $\mathcal{H}=\mathcal{H}^{(0)}+\mathcal{H}^{(1)}+\mathcal{H}^{(2)}+$ $\cdots$, where $\cdots$ means high order terms. The first term in the expansion is $\mathcal{H}^{(0)}=E_{0}=$ $\left(-2 \sqrt{2} t-\mu+\frac{1}{2} n_{0} U\right) N_{0}$, which is the mean-field ground-state energy, then setting $\mathcal{H}^{(1)}=0$ determines chemical potential $\mu=-2 \sqrt{2} t+n_{0} U$ and $E_{0}=-\frac{1}{2} n_{0} U N_{0}$. Diagonalizing $\mathcal{H}^{(2)}$ by a $8 \times 8$ Bogoliubov transformation leads to:

$$
\mathcal{H}^{(2)}=E_{0}^{(2)}+\sum_{\mathbf{q}} \sum_{l=1}^{4} \Omega_{l}(\mathbf{q} ; \phi)\left(\beta_{l, \mathbf{q}}^{\dagger} \beta_{l, \mathbf{q}}+\frac{1}{2}\right)
$$

where $E_{0}^{(2)}=-N_{s}\left(2 \sqrt{2} t+n_{0} U / 2\right)$ and $\mathbf{q} \in\left\{\left(q_{x}, q_{y}\right):\left|q_{x}+q_{y}\right| \leq \pi\right\}$ is the diamond Brillouin zone (BZ) in figure 1(b). ${ }^{1}$ The quantum corrected ground state energy is:

$$
E_{\mathrm{GS}}(\phi)=E_{t 0}+\frac{1}{2} \sum_{\mathbf{q}} \sum_{l=1}^{4} \Omega_{l}(\mathbf{q} ; \phi)
$$

where $E_{t 0}=E_{0}+E_{0}^{(2)}$.

\footnotetext{
${ }^{1}$ In principle, due to the 2 sublattices, 2 valleys, 2 spin, and 2 particle-hole structures, the $16 \times 16$ Bogoliubov transformation leads to 8 bands in the $1 / 2 \mathrm{BZ}$ in figure 1(a). However, we find that we can shift 4 of the 8 bands to make the 4 bands $\Omega_{l}, l=1,2,3,4$ in the (diamond) BZ in figure $1(\mathrm{~b})$.
} 


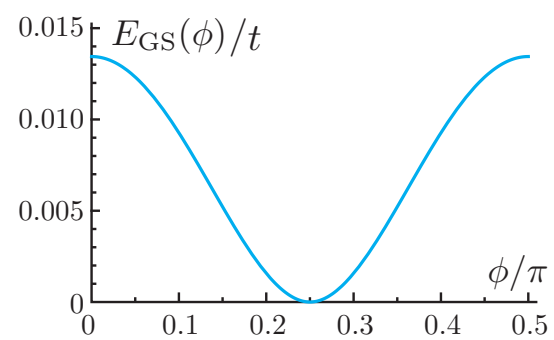

(a)

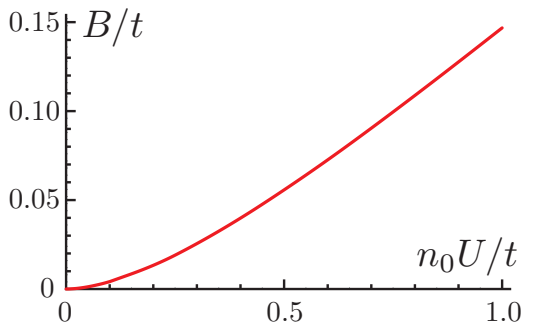

(b)

Figure 3. (a) The quantum ground-state energy $E_{\mathrm{GS}}(\phi)$ has a minimum at $\phi=\pi / 4$. Expanding around the minimum leads to the coefficient $B$ in (b), which is a monotonically increasing function of the interaction strength. In order to clearly see the OFQD, we subtract $E_{\mathrm{GS}}$ by it minimum $\min _{\phi} E_{\mathrm{GS}}$. The parameters are $n_{0} U=t=1$.

The numerical result of $E_{\mathrm{GS}}(\phi)$ as a function of $\phi$ in figure 3(a) shows that $E_{\mathrm{GS}}$ reaches its minima at $\phi=\pi / 4$. Thus, we can expand $E_{\mathrm{GS}}(\phi)$ around its minimum $\phi=\pi / 4+\delta \phi$ as

$$
E_{\mathrm{GS}}\left(\phi, n_{0} U\right)=E_{1}+\frac{B}{2}(\delta \phi)^{2}+\cdots
$$

where $E_{1}=E_{\mathrm{GS}}\left(\phi=\pi / 4, n_{0} U\right)$ and the coefficient $B$ is obtained as

$$
B=\left(n_{0} U\right)^{2} \sum_{\mathbf{q} \in \mathrm{BZ}} \sum_{n=1}^{4} \frac{2^{8} t^{4}\left(\cos q_{x}-\cos q_{y}\right)^{2} \Omega_{n}(\mathbf{q})}{\prod_{m \neq n}\left[\Omega_{n}(\mathbf{q})^{2}-\Omega_{m}(\mathbf{q})^{2}\right]}
$$

where $\Omega_{n}=\Omega_{n}(\mathbf{q}, \phi=\pi / 4)$. We find that $B=c \frac{\left(n_{0} U\right)^{2}}{t}$ where $c \sim a+b \ln (U / t)$ as shown in figure $3(\mathrm{~b})$.

So the "order by disorder" mechanism selects $\phi=\pi / 4$ among the classically degenerate family. Setting $\phi=\pi / 4$ into eq. (2.9) leads to the associated spin structure:

$$
\mathbf{S}_{i}=\frac{1}{4}\left[(-1)^{i_{x}}(1,1,0)+(-1)^{i_{y}}(-1,1,0)\right]
$$

which is a 4-sublattice $90^{\circ}$ spin structure in figure $2(\mathrm{a})$, in comparison with the 3 -sublattice $120^{\circ}$ frustrated spin structure in a triangular lattice [21-23].

\subsection{Excitation spectrum above the $90^{\circ}$ frustrated quantum ground state: a partial contradiction to the Goldstone theorem}

Setting $\phi=\pi / 4$ into the Bogliubov Hamiltonian eq. (2.10), we find that $\Omega_{1}(\mathbf{q})$ contains one linear mode at $(0,0)$ (called $\left.\omega_{1}\right)$ and another at $(\pi, 0)$ (called $\left.\omega_{2}\right) . \Omega_{2}(\mathbf{q})$ contains one quadratic mode at $(0,0)$ (called $\left.\omega_{3}\right)$ and another at $(\pi, 0)\left(\right.$ called $\left.\omega_{4}\right)$. Their expressions can be simplified if we introduce:

$$
\omega_{ \pm}(\mathbf{q})=\sqrt{A_{\mathbf{q}}+B_{\mathbf{q}} \pm \sqrt{4 A_{\mathbf{q}} B_{\mathbf{q}}+C_{\mathbf{q}}^{2}}}
$$


where we define

$$
\begin{aligned}
& A_{\mathbf{q}}=4 t^{2}\left(\cos ^{2} q_{x}+\cos ^{2} q_{y}\right)-\sqrt{2} t n_{0} U\left(\cos q_{x}+\cos q_{y}\right), \\
& B_{\mathbf{q}}=2 t\left(4 t+\sqrt{2} n_{0} U\right), \\
& C_{\mathbf{q}}=\sqrt{2} t n_{0} U\left(4+\cos q_{x}+\cos q_{y}\right),
\end{aligned}
$$

where $\mathbf{q}$ is defined in the $2 \mathrm{BZ}$ in figure $1(\mathrm{~b})$. In the long wavelength limit $\mathbf{q} \rightarrow 0$, the 4 gapless modes can be written as: $\omega_{1}=\omega_{-}(\mathbf{q})=\sqrt{\sqrt{2} t n_{0} U} q, \omega_{2}=\omega_{-}\left(\mathbf{K}_{2}+\mathbf{q}\right)=\omega_{4}=$ $\omega_{-}\left(\mathbf{K}_{4}+\mathbf{q}\right)=\sqrt{\frac{4 t^{2} n_{0} U}{4 \sqrt{2} t+n_{0} U}} q, \omega_{3}=\omega_{-}\left(\mathbf{K}_{3}+\mathbf{q}\right)=\frac{t}{\sqrt{2}} q^{2}$ where $\mathbf{K}_{2}=(\pi, 0), \mathbf{K}_{4}=(0, \pi)$, $\mathbf{K}_{3}=(\pi, \pi){ }^{2}$ Here the degeneracy $\omega_{2}=\omega_{4}$ is dictated by the $\left[C_{4} \times C_{4}\right]_{\text {Diagonal }}$ symmetry of the $90^{\circ}$ state in figure 2(a). One can see that the three linear modes $\omega_{1}, \omega_{2}=\omega_{4}$ are proportional to $\sqrt{n_{0} U t}$, while the quadratic mode $\omega_{3}$ is independent of the interaction $U$. In fact, it is identical to the free particle dispersion. On the other hand, the $90^{\circ}$ coplanar state in figure 2(a) breaks both the $\mathrm{U}(1)_{c}$ and completely the spin $\mathrm{SU}(2)$ symmetry, so it should lead to $1+3$ linear gapless modes instead of 3 linear and 1 quadratic mode. Therefore the quadratic mode $\omega_{3}$ must be an artifact of the Bogliubov calculation to this order. In the following, we show that this in-consistence can be completely resolved by involving the order from quantum disorder mechanism.

\subsection{The slow-Goldstone mode generated by the OFQD mechanism: step 2, a complete path integral formalism of the OFQD}

In the geometrically frustrated quantum spin systems, an "order from quantum disorder" analysis [21-23] was developed to calculate the gap at $\mathbf{k}=0$. When applying this analysis to eq. (2.12), we find that due to the absence of the conjugate $A$ term (as dictated by a $\mathrm{U}(1)$ subgroup of the spin $\mathrm{SU}(2)$ symmetry), there is still no gap at $\mathbf{q}=0$ generated to the quadratic $\omega_{3}$ mode. So the previous analysis in [21-23] can not be used to find any useful information to the present problem. Here, we develop the second step of the systematic "order from disorder analysis" to go beyond that developed in [21-23] to compute the contributions to the spectrum from eq. (2.12). There are two alternative approaches, one is the canonical quantization approach to be presented in detail in appendix A, the other is the boson coherent state path integral approach presented here. The latter is more systematical and powerful than the canonical quantization approach. Most importantly, it can also be used to capture the non-linear effects, so it is essentially a non-linear sigma model approach that can be applied directly to study finite temperature effects. The physical picture is also more transparent in the polar coordinates, especially in identifying the slow-Goldstone mode generated by OFQD.

To capture low-energy excitation above the ground-state, we re-examine eq. (2.3) and eq. (2.4), and study quantum fluctuations rather than just look at the ground-state. In the

\footnotetext{
${ }^{2}$ Note that these $\mathbf{K}_{1,2}$ defined in the $2 \mathrm{BZ}$ are different from its original definitions in the (diamond) BZ in figure $1(\mathrm{~b})$.
} 
$S_{z}$ basis, it is naturally to introduce a parametrization of $\mathbf{z}_{1,2}$

$$
\mathbf{z}_{1}=e^{-i \theta} \cos \phi\left(\begin{array}{c}
e^{-i \alpha_{1} / 2} \cos \left(\beta_{1} / 2\right) \\
e^{+i \alpha_{1} / 2} \sin \left(\beta_{1} / 2\right)
\end{array}\right), \quad \mathbf{z}_{2}=e^{+i \theta} \sin \phi\left(\begin{array}{c}
e^{-i \alpha_{2} / 2} \cos \left(\beta_{2} / 2\right) \\
e^{+i \alpha_{2} / 2} \sin \left(\beta_{2} / 2\right)
\end{array}\right),
$$

then interaction energy density becomes

$$
E_{\text {int }}=\frac{n^{2} U}{2}\left\{1+\frac{1}{2} \sin ^{2}(2 \phi)\left[\cos ^{2}\left(\frac{\alpha_{1}-\alpha_{2}}{2}\right) \cos ^{2}\left(\frac{\beta_{1}-\beta_{2}}{2}\right)+\sin ^{2}\left(\frac{\alpha_{1}-\alpha_{2}}{2}\right) \cos ^{2}\left(\frac{\beta_{1}+\beta_{2}}{2}\right)\right]\right\}
$$

and minimization condition can be expressed as $\alpha_{1}-\alpha_{2}=0$ and $\beta_{1}-\beta_{2}= \pm \pi$.

In the mean-field ground-state manifold, the interaction energy is independent of choice of $\chi, \theta, \alpha_{1}+\alpha_{2}, \beta_{1}+\beta_{2}$ and $\phi$, where the first one is related to $\mathrm{U}(1)_{c}$ symmetry, the second to fourth ones are related to $\mathrm{SU}(2)_{s}$ symmetry, but the last one $\phi$ is due to spurious symmetry. The mean-field ground-state in eq. (2.5) corresponds to choose $\theta=\alpha, \alpha_{1}=-\alpha_{2}=\gamma$, $\beta_{1}=\beta_{2}-\pi / 2=\beta$, and then fix $\chi=\alpha=\beta=\gamma=0$ in eq. (2.7). Here, we prefer to choose a different but equivalent saddle point solution

$$
n=n_{0}, \quad \chi=0, \quad \alpha_{1}=\alpha_{2}=0, \quad \beta_{1}=\beta_{2}+\pi=\pi / 2 .
$$

The corresponding $\mathbf{z}$ spinors are: $\mathbf{z}_{1}=\left(\begin{array}{c}1 / 2 \\ 1 / 2\end{array}\right), \mathbf{z}_{2}=\left(\begin{array}{c}1 / 2 \\ -1 / 2\end{array}\right)$, which are related to those in eq. (2.7) by a $\mathrm{SU}(2)_{s}$ rotation. The former is in the $\sigma_{x}$ representation, the latter is in the $\sigma_{z}$ representation which may be singular in the polar coordinate.

The Bose-Hubbard interaction can be expanded around the saddle point as

$$
\mathcal{H}_{\text {int }}=\frac{U n_{0}^{2}}{2} N_{s}+\sum_{\mathbf{r}} 2 U n_{0} \delta n+\sum_{\mathbf{r}} U(\delta n)^{2}+\sum_{\mathbf{r}} \frac{1}{8} U n_{0}^{2}\left[\left(\delta \beta_{1}-\delta \beta_{2}\right)^{2}+\left(\delta \alpha_{1}-\delta \alpha_{2}\right)^{2}\right] .
$$

Similarly, the Kinetic energy term becomes

$$
\begin{aligned}
\mathcal{H}_{\text {kin }} & =\sum_{\mathbf{k}} \Psi_{\mathbf{k}}^{\dagger}\left(\epsilon_{-}-\mu\right) \Psi_{\mathbf{k}} \\
& \approx-2 \sqrt{2} t n_{0} N_{s}-\mu n_{0} N_{s}-2 \sum_{\mathbf{r}}\left(2 \sqrt{2} t n_{0} N_{s}+\mu\right) \delta n-\sqrt{2} n_{0} t \sum_{\mathbf{r}} \Psi^{\dagger} \nabla^{2} \Psi .
\end{aligned}
$$

Similar to the Bogoliubov theory, the linear term eventually got cancelled by $\mu=-2 \sqrt{2} t+$ $n_{0} U$. After including the boson Berry phase $\Psi^{\dagger} \partial_{\tau} \Psi$ term and order-from-disorder generated potential, we form a complete Lagrangian density

$$
\begin{aligned}
\mathcal{L}_{\mathrm{SF}}= & i \delta n \partial_{\tau} \delta \chi+\frac{i}{4} n_{0}\left(8 \delta \phi \partial_{\tau} \delta \theta+\delta \beta_{1} \partial_{\tau} \delta \alpha_{1}-\delta \beta_{2} \partial_{\tau} \delta \alpha_{2}\right) \\
& -\frac{n_{0} t}{\sqrt{2}}\left[\frac{1}{4 n_{0}^{2}}(\nabla \delta n)^{2}+(\nabla \delta \chi)^{2}+(\nabla \delta \theta)^{2}+(\nabla \delta \phi)^{2}\right] \\
& -\frac{n_{0} t}{8 \sqrt{2}}\left[\left(\nabla \delta \alpha_{1}\right)^{2}+\left(\nabla \delta \alpha_{2}\right)^{2}+\left(\nabla \delta \beta_{1}\right)^{2}+\left(\nabla \delta \beta_{2}\right)^{2}\right] \\
& +\frac{1}{2} U(\delta n)^{2}+\frac{1}{16} n_{0}^{2} U\left[\left(\delta \beta_{1}-\delta \beta_{2}\right)^{2}+\left(\delta \alpha_{1}-\delta \alpha_{2}\right)^{2}\right] \\
& +\frac{B}{2}(\delta \phi)^{2},
\end{aligned}
$$




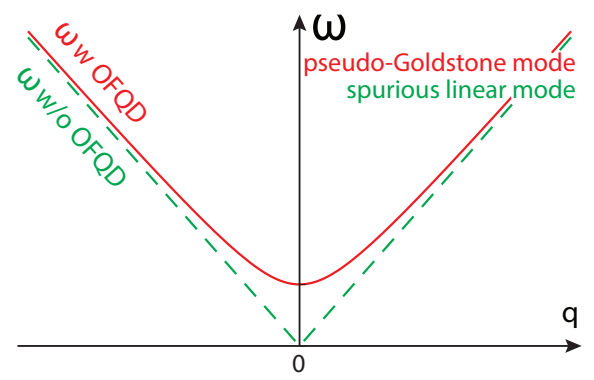

(a)

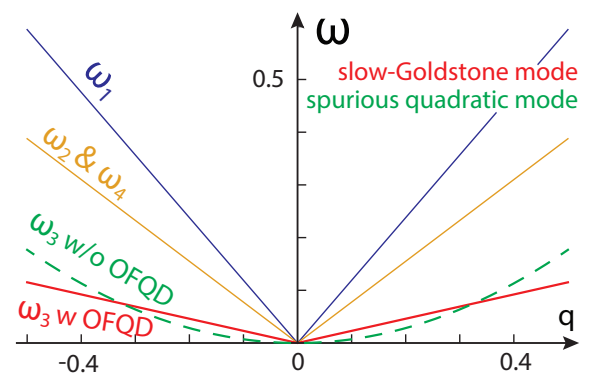

(b)

Figure 4. The pseudo-Goldstone mode discovered in previous works vs slow-Goldstone mode discovered in this work: (a) Typical OFQD generated pseudo-Goldstone mode. The OFQD transfers a spurious linear mode ( $\omega \mathrm{w} /$ o OFQD) into a pseudo-Goldstone mode ( $\omega \mathrm{w}$ OFQD) with a tiny gap. (b) The OFQD generated slow-Goldstone mode $\left(\omega_{3}\right)$ and the other 3 conventional Goldstone modes $\left(\omega_{1,2,4}\right)$. The OFQD transfers a spurious quadratic mode $\left(\omega_{3}\right.$ w/o OFQD cf. eq. (2.15)) into a slow-Goldstone mode $\left(\omega_{3} \mathrm{w}\right.$ OFQD cf. eq. (2.25)) with a very small velocity.

where the last term is the order-from-disorder generated potential. The Lagrangian density contains four conjugate pairs: $(\delta n, \delta \chi),(\delta \phi, \delta \theta),\left(\delta \beta_{1}, \delta \alpha_{1}\right),\left(\delta \beta_{2}, \delta \alpha_{2}\right)$. The conjugate pair $(\delta n, \delta \chi)$ is density fluctuations and global phase fluctuations, which gives superfluid sound mode; in contrast, the other three pairs contribute to the spin fluctuations, which give the three spin modes. The conjugate pair $(\delta \phi, \delta \theta)$ is associated with the spurious symmetry. Before considering the order from quantum disorder effect, $(\delta \phi, \delta \theta)$ behaves just like two transverse components of the magnetization in a ferromagnet, which results in a "spurious" quadratic dispersion $\omega \sim q^{2}$. However, after including the order from quantum disorder effect, $\delta \phi$ acquires a small finite OfD generated potential, thus it modifies the quadratic dispersion to a true linear one $\omega \sim q$.

Finally, In the long-wave length limit, these four conjugate pairs give 4 linear gapless modes:

$$
\begin{aligned}
& \omega_{1}=v_{1} q=\sqrt{\sqrt{2} t n_{0} U} q, \\
& \omega_{2}=\omega_{4}=v_{2} q=\sqrt{\frac{4 t^{2} n_{0} U}{4 \sqrt{2} t+n_{0} U}} q \sim \omega_{1} / \sqrt{2} \\
& \omega_{3}=v_{3} q=\sqrt{\frac{B t}{2 \sqrt{2} n_{0}}} q
\end{aligned}
$$

where $q=|\mathbf{q}|=\sqrt{q_{x}^{2}+q_{y}^{2}}$. Especially, we find $(\theta, \phi)$ conjugate pair in eq. (2.19) is the slowGoldstone mode generated by OFQD. As a comparison, we illustrate the OFQD generated pseudo-Goldstone mode and OFQD generated slow-Goldstone mode in figure 4(a) and (b), respectively. Using the fact $B=c\left(n_{0} U\right)^{2} / t$, its velocity $v_{3} \sim n_{0}^{1 / 2} U$ vanishes in the $U \rightarrow 0$ limit. In the weak coupling limit, $U / t \ll 1$, we have relation

$$
v_{1}>v_{2}=v_{4} \sim v_{1} / \sqrt{2} \gg v_{3}
$$




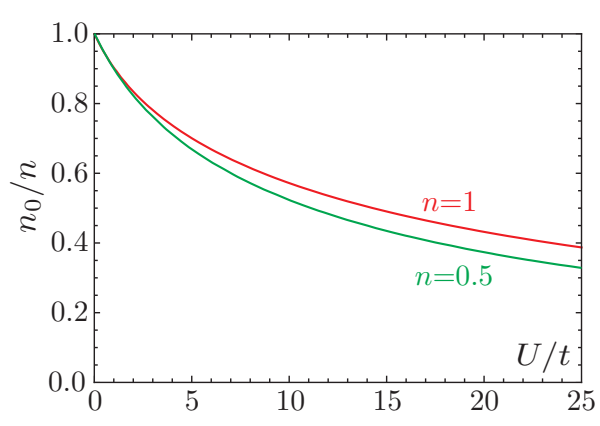

(a)

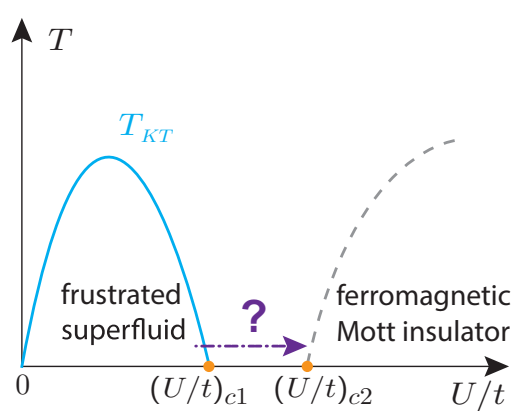

(b)

Figure 5. (a) The condensate fraction $n_{0} / n$ as a function of $U / t$ when filling factor $n=0.5$ and $n=1$. (b) At $T=0$, there could be some kind of quantum spin liquid (QSL) intervening between the frustrated SF at weak coupling and the FM SF at strong coupling.

So the $\omega_{3}$ mode becomes linear only in a tiny regime near zero momentum, beyond which it recovers the quadratic free particle mode. The $\omega_{3}$ mode is the slow-Goldstone mode generated by the order from quantum disorder. Because the slow-Goldstone mode is much softer than the other three Goldstone modes, it can be easily identified in the spinor cold atom or photonic experiments.

\subsection{The condensate fraction and the quantum depletions}

From eq. (2.10), (2.15), (2.18), we can evaluate the quantum depletion $N_{1}=N-N_{0}$ :

$$
N_{1}=\frac{1}{4} \sum_{\mathbf{q}, s= \pm}\left[\frac{1}{\omega_{s}(\mathbf{q})}\left(4 \sqrt{2} t+n_{0} U+s \frac{2\left(4 \sqrt{2} t+n_{0} U\right) A_{\mathbf{q}}+n_{0} U C_{\mathbf{q}}}{\sqrt{4 A_{\mathbf{q}} B_{\mathbf{q}}+C_{\mathbf{q}}^{2}}}\right)-1\right]
$$

where sum over $\mathbf{q}$ is restricted to the $2 \mathrm{BZ}$ in figure $1(\mathrm{~b})$.

In the cold atom experiments, the total density $n$ is given. Then the condensate density $n_{0}$ can be solved with a fixed values of $n$ and $U$ in eq. (2.27). Shown in figure 5(a) is the condensate fraction $n_{0} / n$ as a function of $n$ and $U$. where one can see at $U / t \ll 1$, $1-n_{0} / n \ll 1$ justifies the perturbation theory in the weak coupling limit. As $U / t$ gets larger, at integer fillings $n$, the system remains in the SF state when $U / t$ is below a critical value [figure 5(b)], the ground state remains the 4 -sublattice coplanar $90^{\circ}$ state in figure 3 with the 4 gapless linear modes. However, their velocities in eq. (2.25) may not be precise anymore, when near $(U / t)_{c}$.

\subsection{The strong coupling ferromagnetic Mott state}

In the strong coupling limit $U / t \gg 1$, at the integer fillings, the system gets into a Mott insulating state. The second order perturbation in the strong coupling expansion in $t^{2} / U$ lead to a spin- $n / 2$ Ferromagnetic Heisenberg model [42]:

$$
\mathcal{H}_{\text {spin }}=-J \sum_{\langle i j\rangle} \mathbf{S}_{i} \cdot \mathbf{S}_{j}
$$


where $J=4 t^{2} / U$. Note that the 0 or $\pi$ flux makes no difference in the strong coupling limit to this order.

So the ground state in the strong coupling limit is nothing but the FM Mott state. The dispersion in the long wavelength limit is the well-known quadratic gapless FM mode

$$
\omega_{\mathrm{FM}}(k)=\frac{n t^{2}}{U}\left(k_{x}^{2}+k_{y}^{2}\right),
$$

which is due to the spontaneous symmetry breaking $\mathrm{SU}(2)_{s} \rightarrow \mathrm{U}(1)_{s}$. This is in sharp contrast to the symmetry breaking $\mathrm{U}(1)_{c} \times \mathrm{SU}(2)_{s} \rightarrow 1$ leading to 4 linear Goldstone modes in the frustrated SF phase in eq. (2.25). Note that the FM state included in the classically degenerate family of states at the weak coupling in figure 2(c) is a SF state instead of a Mott state.

As shown in the previous paragraph, at weak coupling, there is a BEC $\rightarrow$ superfluid at $T=0$, then the $\mathrm{U}(1)_{c} \times \mathrm{SU}(2)_{s} \rightarrow 1$ is completely broken, which leads to the 4 Goldstone modes, and one of which is the slow-Goldstone mode. There is also an associated lattice symmetry breaking shown in figure 2. At any finite $T$, the continuous symmetries have to be restored, so only one of the 4 Goldstone modes: the superfluid one $\omega_{1}$ survives. The finite temperature phase transition at weak coupling could be just the Kosterlitz-Thouless (KT) transition [figure 5(b)]. Of course, the FM order at strong coupling also disappears at any finite $T$. The outstanding problem remaining is to find what could be the intervening phase between the two limits in figure 5(b). This will be addressed in the following work [41].

\section{The implications on cold atom and photonic experiments}

In fact, the problem of a particle moving in a lattice subject to an Abelian flux has a long history. The most original system is free electrons moving in a solid subject to a magnetic flux $\alpha$ through a unit cell which is called Hofstadter problem [43-45]. Unfortunately, it needs an astronomical large magnetic field to generate any appreciable magnetic flux $\alpha$ through each unit cell of a solid, so it is difficult to realize the Hofstadter problem of free electrons in any solid (However, for a recent progress in graphene, see [46]). Fortunately, there are recent experimental advances to generate effective magnetic flux $\alpha$ through each unit cell of an optical lattice for cold atoms [27-36] and that of a microwave cavity array for photons [37-40]. Both the cold atom and the photonic systems lead to the bosonic analog of Hofstadter problem. Of course, in contrast to fermions, bosons are necessarily interacting, so one must incorporate interactions onto the problem.

On the other hand, it was well known the cold atom systems in an optical lattice suffer the heating problem. Fortunately, it is still well under control with enough long lifetime at weak coupling, but gets worse as the coupling strength increase. So the frustrated SF at the weak couplings in figure 5 can be directly probed in the current cold atom experiments, but the FM may not (at this stage). In this section, we only focus on the experimental detections in the weak coupling regime.

The cold atom condensation wave-function can be directly imaged through time-offlight (TOF) images [47], which after a time $t$ is given by:

$$
n(\mathbf{r})=(M / \hbar t)^{3} f(\mathbf{k}) G(\mathbf{k}),
$$


where $\mathbf{k}=M \mathbf{r} / \hbar t, f(\mathbf{k})=|w(\mathbf{k})|^{2}$ is the form factor due to the Wannier state of the lowest Bloch band of the optical lattice, and $G(\mathbf{k})=\frac{1}{N_{s}} \sum_{i, j} e^{-\mathbf{k} \cdot\left(\mathbf{r}_{i}-\mathbf{r}_{j}\right)}\left\langle\Psi_{i}^{\dagger} \Psi_{j}\right\rangle$ is the equal time boson structure factor. For a small condensate depletion shown in figure 5(a) $\left\langle\Psi_{i}^{\dagger} \Psi_{j}\right\rangle \sim\left\langle\Psi_{0 i}^{\dagger} \Psi_{0 j}\right\rangle$, where $\Psi_{0 i}^{\dagger}$ is the condensate wave-function eq. (2.7) at $\phi=\pi / 4$. So the TOF can detect the quantum ground state wave-function directly. The quantum depletion calculated in eq. (2.27) leads to a reduction in the magnitude of the condensation peaks at $\mathbf{K}$ and $-\mathbf{K}$ in eq. (2.7) and some broad backgrounds.

The 4 -sublattice $90^{\circ}$ spin-orbital structure eq. (2.14) in figure 3, the 4 gapless modes in eq. (2.25) and the nature of the transition in figure $5(\mathrm{~b})$ can be precisely determined by dynamic or elastic, energy or momentum resolved, longitudinal or transverse Bragg spectroscopies [48-60] in cold atoms and the site- and time-resolved spectroscopy [37-40] in photonic systems. As stressed below eq. (2.25), the Goldstone mode $\omega_{3}$ generated by OFQD is much softer than the other three Goldstone modes, so it can be easily distinguished by these experimental techniques. Notably, both Goldstone and Higgs modes were detected near the two-dimensional superfluid/Mott insulator transition [61] and in a supersolid cold atomic quantum gas [62].

\section{Discussion and conclusion}

The OFQD was first discovered in the geometrically frustrated magnets [21-23]. However, so far, it was developed only to find the quantum ground state and then calculate the gap generated by the phenomena, but not the spectrum yet. Here, we developed a new and systematic two-step OFQD analysis to calculate not only the mass gap but also the whole excitation spectrum. This systematic development, especially its second step, is vital to discover the slow-Goldstone mode generated by the OFQD. The new method developed in this work can be transformed to compute the whole excitation spectrum in all the other frustrated systems such as geometric frustrated systems, a system subject to an non-Abelain flux and particle physics. The novel phenomenon of the slow-Goldstone mode generated by OFQD may also appear in these quantum systems.

As mentioned in the introduction, there may be also an associated spurious Goldstone mode resulting from the breaking of a spurious continuous symmetry. Then quantum fluctuations may generate a small gap to this spurious Goldstone mode and transfer it into a pseudo-Goldstone mode. In particle physics, there is a closely related phenomena called the Coleman-Weinberg effective potential [17]. For example, the pion with a light mass maybe just such a pseudo-Goldstone mode $[18,19]$. The putative axion with a tiny mass maybe also such a pseudo-Goldstone mode [63-65] with the approximate global (Peccei-Quinn) U(1) symmetry corresponding to the spurious U(1) symmetry here. Here we discover a completely opposite new phenomenon: a true slow-Goldstone mode generated by the OFQD, which can be observed in the current spinor cold atom or photonic experiments subject to an Abelian $\alpha=\pi$ flux. It is important to explore possible connections between the effective potential generated by the OFQD presented here and the Coleman-Weinberg potential in relativistic quantum field theory. It is also interesting to see if this opposite new phenomenon may also happen in particle physics. 
It is instructive to look at the OFQD from the entropy point of view. At the classical level, the spurious U(1) symmetry leads to a classically degenerate family of ground-states which, in turn, leads to an extensive ground state degeneracy, therefore an extensive $T=0$ entropy, clearly violating the third law of thermodynamics. However, as shown here, the OFQD spoils the spurious U(1) symmetry, picks a unique quantum ground state, therefore leads to a vanishing the $T=0$ entropy, consistent with the third law of thermodynamics. In the SYK models [6-9], at the mean-field level $N=\infty$, there is also an extensive ground state degeneracy, therefore the extensive $T=0$ entropy $s_{0} N$ with $s_{0}=0.2324 \ldots$, clearly violating the third law of thermodynamics. However, the $1 / N$ expansion picks a unique quantum ground state, therefore leads to a vanishing the $T=0$ entropy consistent with the third law of thermodynamics. It was argued that the $T=0$ entropy $s_{0}$ corresponds to the classical Bekenstein-Hawking (BH) entropy of a classical black hole in the AdS bulk. It remains interesting to see if the quantum fluctuations will drive to classical BH entropy for an extreme black hole at $T=0$ to zero as dictated by the third law of thermodynamics. However, the main difference between condensed matter or cold atom systems and quantum black holes is the causality: no horizon in the former separates the timelike from the spacelike. The black hole has a horizon which is equal to its area, so the third law of thermodynamics may need to be modified to consider this fact: its entropy for an extreme black hole at $T=0$ may still be non-zero because an observer outside the black hole can not see what is inside the black hole, the most he/she can see is the surface area of the black hole. Then one may need to modify the third law of thermodynamics to: the entropy of an extreme black hole at $T=0$ reaches its minimum, which is the surface of the black hole, namely, the BH entropy. If one squeezes the surface area to a point, then the BH entropy becomes zero, and one recovers the third law of thermodynamics. It remains outstanding to evaluate the quantum corrections to the classical $\mathrm{BH}$ entropy for the extreme black holes, for example, in the context of $2 \mathrm{~d}$ quantum gravity, which is dual to SYK models. For a non-extreme black hole at any finite $T$, then one may start to consider its Hawking radiations.

Note added. In a recent work [41], we show that there is indeed a $Z_{2}$ QSL intervening between the frustrated SF at weak coupling and the FM Mott at strong coupling in figure 5(b). So this work focus on symmetry broken states and the associated gapless excitations. The approach used here is the microscopic calculations at both weak and strong couplings. While [41] focus on topological states and the associated gapped fractionalized topological excitations. The approach to be used there is a symmetry based effective action at any coupling strengths. We will also establish the intrinsic relations between the two complementary approaches.

\section{Acknowledgments}

The authors thank Prof. Dapeng Yu for hospitality during the authors' visit at Institute for Quantum Science and Engineering, Shenzhen, China, and thank Prof. Wei Ku for the hospitality during the authors visit at the Tsung-Dao Lee Institute, Shanghai, China. This research was supported by AFOSR FA9550-16-1-0412. 


\section{A The second step of the newly developed systematic "order from quan- tum disorder analysis" to compute the spectra of the slow-Goldstone mode: canonical quantization approach}

In the appendix A, we provide some technical details on the second step of the newly developed systematic "order from quantum disorder analysis" to compute the spectrum of the slow-Goldstone mode by the canonical quantization approach, which is complementary to the boson coherent state path integral approach developed in the main text. Reaching the same set of Goldstone modes by two independent and complementary approaches not only confirm the correctness of the results, but also provide additional physical insights into the novel phenomenon of the slow-Goldstone mode generated by the OFQD.

In order to compute the corrections to the excitation spectrum due to the OFQD phenomena in the canonical quantization approach, one need to express the order from disorder field operator $\delta \phi_{\mathbf{r}}$ in eq. (2.12) in terms of the original boson field operator in eq. (2.1). The most generic state including all the possible quantum fluctuations are parameterized in eq. (2.5). The $90^{\circ}$ quantum ground state corresponds to its saddle point value $\Psi_{0}=\left(\rho_{0}, 0, \pi / 4,0, \chi_{1 c}, \chi_{2 c}\right)$. One can write down the most generic quantum fluctuations around the saddle point as

$$
\Psi_{i}=\Psi_{i, 0}+\left(\delta \rho, \theta, \delta \phi, \alpha, \delta \chi_{1}, \delta \chi_{2}\right)
$$

The Bose field can be separated into the condensation part plus the quantum fluctuation part in a polar-like coordinate system:

$$
\Psi_{\mathbf{r}}=\Psi_{\mathbf{r}, 0}+\delta \Psi_{1} e^{-i \mathbf{K} \cdot \mathbf{r}}+\delta \Psi_{2} e^{i \mathbf{K} \cdot \mathbf{r}},
$$

where the components at $-\mathbf{K}$ and $\mathbf{K}$ are:

$$
\begin{aligned}
& \delta \Psi_{1}=\frac{\delta \rho-2 \rho_{0} \delta \phi+i 2 \rho_{0}(\theta+\alpha)}{2 \sqrt{\rho_{0}}}\left(\eta_{1} \otimes \chi_{1 c}\right)+\sqrt{\rho_{0}}\left(\eta_{1} \otimes \delta \chi_{1}\right) \\
& \delta \Psi_{2}=\frac{\delta \rho+2 \rho_{0} \delta \phi+i 2 \rho_{0}(\theta-\alpha)}{2 \sqrt{\rho_{0}}}\left(\eta_{2} \otimes \chi_{2 c}\right)+\sqrt{\rho_{0}}\left(\eta_{2} \otimes \delta \chi_{2}\right)
\end{aligned}
$$

After comparing it with the decomposition in the Cartesian coordinate, using the fact $\operatorname{Re}\left[\chi_{1 c}^{\dagger} \cdot \delta \chi_{1}\right]=\operatorname{Re}\left[\chi_{2 c}^{\dagger} \cdot \delta \chi_{2}\right]=0$ and introducing the $\xi_{i}, i=1,2$ to be othor-normal to $\eta_{i}$, namely $\xi_{i}^{\dagger} \eta_{j}=\delta_{i j}$, One can express the order from disorder variable $\delta \phi_{\mathbf{r}}$ in terms of the original Bose fluctuation field:

$$
\begin{aligned}
\delta \phi_{q}=[ & -\bar{\xi}_{1 a} \delta a_{\mathbf{K}+\mathbf{q} \uparrow}-\xi_{1 a} \delta a_{\mathbf{K}-\mathbf{q} \uparrow}^{\dagger}+\bar{\xi}_{2 a} \delta a_{-\mathbf{K}+\mathbf{q} \downarrow}+\xi_{2 a} \delta a_{-\mathbf{K}-\mathbf{q} \downarrow}^{\dagger} \\
& \left.-\bar{\xi}_{1 b} \delta b_{\mathbf{K}+\mathbf{q} \uparrow}-\xi_{1 b} \delta b_{\mathbf{K}-\mathbf{q} \uparrow}^{\dagger}+\bar{\xi}_{2 b} \delta b_{-\mathbf{K}+\mathbf{q} \downarrow}+\xi_{2 b} \delta b_{-\mathbf{K}-\mathbf{q} \downarrow}^{\dagger}\right] /\left(4 \sqrt{\rho_{0}}\right),
\end{aligned}
$$

where as expected, both the quantum fluctuations near $-\mathbf{K}$ and $\mathbf{K}$ in the Cartesian coordinate appear in the right side of the equation. Thus we can express the quantum corrections from the OFQD in terms of the original Bose fields as

$$
\delta \mathcal{H}=\frac{B}{2} \sum_{q} \delta \phi_{q} \delta \phi_{-q}
$$


Finally, after combining eq. (A.5) with the $\mathcal{H}^{(2)}$ in eq. (2.10), <we arrive at the effective Hamiltonian incorporating the order from quantum disorder (OFQD) mechanism:

$$
H_{\mathrm{OFD}}=H^{(2)}+\delta H=\frac{1}{2} \sum_{q} \Psi_{q}^{\dagger}(M+\delta M) \Psi_{q}
$$

where the $\delta M$ can be written as the following $8 \times 8$ matrix.

$$
\delta M=\frac{B}{16 n_{0}}\left(\begin{array}{cccccccc}
1 & 1 & e^{-i \pi / 4} & e^{i \pi / 4} & -1 & -1 & -e^{i \pi / 4} & -e^{-i \pi / 4} \\
1 & 1 & e^{-i \pi / 4} & e^{i \pi / 4} & -1 & -1 & -e^{i \pi / 4} & -e^{-i \pi / 4} \\
e^{i \pi / 4} & e^{i \pi / 4} & 1 & i & -e^{i \pi / 4} & -e^{i \pi / 4} & -i & -1 \\
e^{-i \pi / 4} & e^{-i \pi / 4} & -i & 1 & -e^{-i \pi / 4} & -e^{-i \pi / 4} & -1 & i \\
-1 & -1 & -e^{-i \pi / 4} & -e^{i \pi / 4} & 1 & 1 & e^{i \pi / 4} & e^{-i \pi / 4} \\
-1 & -1 & -e^{-i \pi / 4} & -e^{i \pi / 4} & 1 & 1 & e^{i \pi / 4} & e^{-i \pi / 4} \\
-e^{-i \pi / 4} & -e^{-i \pi / 4} & i & -1 & e^{-i \pi / 4} & e^{-i \pi / 4} & 1 & -i \\
-e^{i \pi / 4} & -e^{i \pi / 4} & -1 & -i & e^{i \pi / 4} & e^{i \pi / 4} & i & 1
\end{array}\right)
$$

After applying Bogoliubov transformation to eq. (A.7), we find the order from disorder correction eq. (A.5) transfers the quadratic dispersion $\Omega_{2}(\mathbf{q})=\omega_{3}(\mathbf{q})=\frac{t}{\sqrt{2}} q^{2}$ into a linear one $\omega_{3}=v_{3} q=\sqrt{\frac{B t}{2 \sqrt{2} n_{0}}} q$. We also found that the SF Goldstone mode $\Omega_{1}(\mathbf{q})=$ $\omega_{1}(\mathbf{q})=v_{1} q$ remains the same. Due to the momenta separation, $\Omega_{1}((\pi, 0)+\mathbf{q})=\omega_{2}(\mathbf{q})$ and $\Omega_{2}((\pi, 0)+\mathbf{q})=\omega_{4}(\mathbf{q})$ are not affected by the OFQD analysis. All the 4 linear modes were listed in eq. (2.25).

\section{B The kinetic energies and currents in the 4 -sublattice $90^{\circ}$ coplanar ground state}

In the appendix $\mathrm{B}$, we evaluate the kinetic energy and current in the 4 -sublattice $90^{\circ}$ coplanar quantum ground state.

Using the method in $[66,67]$, we will evaluate the conserved density-currents in the 4 sublattice $90^{\circ}$ coplanar ground state in figure $2(\mathrm{a})$. In the context of $2 \mathrm{~d}$ charge-vortex duality $[66,67]$, the vortex currents in the dual lattice gives the boson densities in the direct lattice. Here, we evaluate them directly on the direct lattice.

We can write the kinetic energy in the unit cell in figure 1(a) as:

$$
\mathcal{H}_{\text {hop }}=\sum_{\mathbf{r}}\left[H_{0}+H_{1}+H_{2}+H_{3}+\text { h.c. }\right]
$$

where $H_{0}=t a_{\mathbf{r} \sigma}^{\dagger} b_{\mathbf{r} \sigma}, H_{1}=-t a_{\mathbf{r} \sigma}^{\dagger} b_{\mathbf{r}+\mathbf{v}_{1}, \sigma}, H_{2}=-t a_{\mathbf{r} \sigma}^{\dagger} b_{\mathbf{r}+\mathbf{v}_{2}, \sigma}, H_{3}=-t a_{\mathbf{r} \sigma}^{\dagger} b_{\mathbf{r}+\mathbf{v}_{1}+\mathbf{v}_{2}, \sigma}$ and the two lattice vectors in figure 1 (a) are $\mathbf{v}_{1}=(1,1), \mathbf{v}_{2}=(-1,1)$. Obviously, in the $Z_{2}$ gauge, the first bond is frustrated, the other three are not. Along a given bond $(i, i+\mu)$ :

$$
H_{\mu}=K_{\mu}-i I_{\mu}
$$

where $K$ is the kinetic energy and $I$ is the current flowing along the bond $[66,67]$. 
The mean-field wave-function of the 4 -sublattice $90^{\circ}$ coplanar ground state is given in eq. (2.7) with $\phi=\pi / 4$ and $\mathbf{r}$ labels the unit cell which contains both $A$ and $B$ sublattice sites. Since any nearest neighbor inside a unit cell consists of A-B sublattice sites, we only need to evaluate the form $a_{\mathbf{r}}^{\dagger} b_{\mathbf{r}+\boldsymbol{\mu}}$. Substituting the wave-function into the form leads to:

$$
a_{\mathbf{r}}^{\dagger} b_{\mathbf{r}+\mu}=-\operatorname{Re}\left[n_{0} e^{i \frac{\pi}{4}} e^{i \mathbf{K} \cdot \boldsymbol{\mu}}\right]
$$

which simplifies to

$$
H_{0}=H_{1}=H_{2}=H_{3}=-n_{0} t / \sqrt{2}
$$

It shows that the currents vanish and the kinetic energies are uniform in the ground state. If the system has open edges, then there is no edge states. The system just arranges itself into the 4-sublattice $90^{\circ}$ coplanar ground state to offset the external $\pi$ flux and lead to no currents and no frustrated kinetic energy bonds. If the system has edges, then there is no edge currents flowing along the edges.

Open Access. This article is distributed under the terms of the Creative Commons Attribution License (CC-BY 4.0), which permits any use, distribution and reproduction in any medium, provided the original author(s) and source are credited.

\section{References}

[1] Y. Nambu, Quasiparticles and Gauge Invariance in the Theory of Superconductivity, Phys. Rev. 117 (1960) 648 [INSPIRE].

[2] J. Goldstone, Field Theories with Superconductor Solutions, Nuovo Cim. 19 (1961) 154 [INSPIRE].

[3] J. Goldstone, A. Salam and S. Weinberg, Broken Symmetries, Phys. Rev. 127 (1962) 965 [INSPIRE].

[4] K. Becker, M. Becker and J.H. Schwarz, String Theory and M-Theory: A Modern Introduction, Cambridge University Press (2006), [DOI].

[5] E. Kiritsis, String Theory in a Nutshell, 1st edition, Cambridge University Press (2007).

[6] S. Sachdev and J. Ye, Gapless spin fluid ground state in a random, quantum Heisenberg magnet, Phys. Rev. Lett. 70 (1993) 3339 [cond-mat/9212030] [INSPIRE].

[7] A. Kitaev, A simple model of quantum holography (part 1), talk at KITP, April 7, 2015, http://online.kitp.ucsb.edu/online/entangled15/kitaev/.

[8] A. Kitaev, A simple model of quantum holography (part 2), talk at KITP, May 27, 2015, http://online.kitp.ucsb.edu/online/entangled15/kitaev2/.

[9] J. Maldacena and D. Stanford, Remarks on the Sachdev-Ye-Kitaev model, Phys. Rev. D 94 (2016) 106002 [arXiv:1604.07818] [INSPIRE].

[10] M.E. Peskin and D.V. Schroeder, An introduction to quantum field theory, Taylor \& Francis Inc. eds., Boulder Colorado (1995).

[11] S. Sachdev and J. Ye, Universal quantum critical dynamics of two-dimensional antiferromagnets, Phys. Rev. Lett. 69 (1992) 2411 [cond-mat/9204001] [INSPIRE]. 
[12] A.V. Chubukov, S. Sachdev and J. Ye, Theory of two-dimensional quantum Heisenberg antiferromagnets with a nearly critical ground state, Phys. Rev. B 49 (1994) 11919 [cond-mat/9304046] [INSPIRE].

[13] J. Ye and C. Zhang, Superradiance, Berry phase, photon phase diffusion, and number squeezed state in the U(1) Dicke (Tavis-Cummings) model, Phys. Rev. A 84 (2011) 023840.

[14] Y.-X. Yu, J. Ye and W.-M. Liu, Goldstone and Higgs modes of photons inside a cavity, Sci. Rep. 3 (2013) 3476.

[15] Y. Yi-Xiang, J. Ye and C. Zhang, Parity oscillations and photon correlation functions in the $Z_{2}-\mathrm{U}(1)$ Dicke model at a finite number of atoms or qubits, Phys. Rev. A 94 (2016) 023830.

[16] B. Zeng, X. Chen, D.-L. Zhou and X.-G. Wen, Quantum Information Meets Quantum Matter, Springer-Verlag New York (2019), [DOI].

[17] S.R. Coleman and E.J. Weinberg, Radiative Corrections as the Origin of Spontaneous Symmetry Breaking, Phys. Rev. D 7 (1973) 1888 [INSPIRE].

[18] S. Weinberg, Approximate symmetries and pseudoGoldstone bosons, Phys. Rev. Lett. 29 (1972) 1698 [INSPIRE].

[19] S. Weinberg, The Quantum Theory of Fields, vol. 2, Cambridge University Press (1996), [DOI].

[20] S. Sachdev, Kagome- and triangular-lattice heisenberg antiferromagnets: Ordering from quantum fluctuations and quantum-disordered ground states with unconfined bosonic spinons, Phys. Rev. B 45 (1992) 12377.

[21] G. Murthy, D. Arovas and A. Auerbach, Superfluids and supersolids on frustrated two-dimensional lattices, Phys. Rev. B 55 (1997) 3104.

[22] C. Lhuillier and G. Misguich, Frustrated quantum magnets, (2001) [cond-mat/0109146].

[23] S. Sachdev, Quantum Phase Transitions, Cambridge University Press, 2nd ed. (2011), [DOI].

[24] S. Sachdev and N. Read, Large $n$ expansion for frustrated and doped quantum antiferromagnets, Int. J. Mod. Phys. B 05 (1991) 219.

[25] L. Balents, Spin liquids in frustrated magnets, Nature 464 (2010) 199.

[26] C. Lacroix, P. Mendels and F. Mila, Introduction to Frustrated Magnetism: Materials, Experiments, Theory, Springer-Verlag Berlin Heidelberg (2011), [DOI].

[27] M. Aidelsburger, M. Atala, S. Nascimbène, S. Trotzky, Y.-A. Chen and I. Bloch, Experimental realization of strong effective magnetic fields in an optical lattice, Phys. Rev. Lett. 107 (2011) 255301.

[28] J. Struck et al., Quantum simulation of frustrated classical magnetism in triangular optical lattices, Science $\mathbf{3 3 3}$ (2011) 996

[https://science.sciencemag.org/content/333/6045/996.full.pdf].

[29] J. Struck et al., Tunable gauge potential for neutral and spinless particles in driven optical lattices, Phys. Rev. Lett. 108 (2012) 225304.

[30] J. Struck et al., Engineering Ising-XY spin-models in a triangular lattice using tunable artificial gauge fields, Nature Phys. 9 (2012) 738. 
[31] M. Aidelsburger, M. Atala, M. Lohse, J.T. Barreiro, B. Paredes and I. Bloch, Realization of the Hofstadter Hamiltonian with Ultracold Atoms in Optical Lattices, Phys. Rev. Lett. 111 (2013) 185301.

[32] H. Miyake, G.A. Siviloglou, C.J. Kennedy, W.C. Burton and W. Ketterle, Realizing the harper hamiltonian with laser-assisted tunneling in optical lattices, Phys. Rev. Lett. 111 (2013) 185302.

[33] C.J. Kennedy, G.A. Siviloglou, H. Miyake, W.C. Burton and W. Ketterle, Spin-orbit coupling and quantum spin hall effect for neutral atoms without spin flips, Phys. Rev. Lett. 111 (2013) 225301.

[34] M. Atala, M. Aidelsburger, M. Lohse, J.T. Barreiro1, B. Paredes and I. Bloch, Observation of chiral currents with ultracold atoms in bosonic ladders, Nature Phys. 10 (2014) 588.

[35] M. Aidelsburger et al., Measuring the Chern number of Hofstadter bands with ultracold bosonic atoms, Natue Phys. 11 (2015) 16 [arXiv:1407.4205].

[36] G. Jotzu et al., Experimental realization of the topological Haldane model with ultracold fermions, Nature 515 (2014) 237.

[37] C. Owens et al., Quarter-flux Hofstadter lattice in a qubit-compatible microwave cavity array, Phys. Rev. A 97 (2018) 013818.

[38] B.M. Anderson, R. Ma, C. Owens, D.I. Schuster and J. Simon, Engineering topological many-body materials in microwave cavity arrays, Phys. Rev. X 6 (2016) 041043.

[39] J. Ningyuan, C. Owens, A. Sommer, D. Schuster and J. Simon, Time- and site-resolved dynamics in a topological circuit, Phys. Rev. X 5 (2015) 021031.

[40] N. Schine, A. Ryou, A. Gromov, A. Sommer and J. Simon, Synthetic Landau levels for photons, Nature 534 (2016) 671.

[41] F. Sun and J. Ye, Quantum spin liquids in a square lattice subject to an abelian flux and its experimental observation, (2019) [arXiv:2005.04695].

[42] F. Sun, J. Ye, W.-M. Liu, J. Ye and W.-M. Liu, Quantum magnetism of spinor bosons in optical lattices with synthetic non-Abelian gauge fields, Phys. Rev. A 92 (2015) 043609 [arXiv: 1408.3399] [INSPIRE].

[43] D.R. Hofstadter, Energy levels and wave functions of Bloch electrons in rational and irrational magnetic fields, Phys. Rev. B 14 (1976) 2239 [INSPIRE].

[44] J. Zak, Magnetic Translation Group, Phys. Rev. 134 (1964) A1602 [InSPIRE].

[45] J. Zak, Magnetic translation group. ii. irreducible representations, Phys. Rev. 134 (1964) A1607.

[46] C.R. Dean et al., Hofstadter's butterfly and the fractal quantum hall effect in moiré superlattices, Nature 497 (2013) 598.

[47] I. Bloch, J. Dalibard and W. Zwerger, Many-body physics with ultracold gases, Rev. Mod. Phys. 80 (2008) 885 [arXiv:0704.3011] [INSPIRE].

[48] M. Kozuma et al., Coherent splitting of bose-einstein condensed atoms with optically induced bragg diffraction, Phys. Rev. Lett. 82 (1999) 871.

[49] J. Stenger, S. Inouye, A.P. Chikkatur, D.M. Stamper-Kurn, D.E. Pritchard and W. Ketterle, Bragg spectroscopy of a bose-einstein condensate, Phys. Rev. Lett. 82 (1999) 4569. 
[50] D.M. Stamper-Kurn et al., Excitation of phonons in a bose-einstein condensate by light scattering, Phys. Rev. Lett. 83 (1999) 2876.

[51] J. Steinhauer, R. Ozeri, N. Katz and N. Davidson, Excitation spectrum of a bose-einstein condensate, Phys. Rev. Lett. 88 (2002) 120407.

[52] S.B. Papp et al., Bragg spectroscopy of a strongly interacting ${ }^{85} \mathrm{Rb}$ bose-einstein condensate, Phys. Rev. Lett. 101 (2008) 135301.

[53] P.T. Ernst et al., Probing superfluids in optical lattices by momentum-resolved bragg spectroscopy, Nature Phys. 6 (2009) 56.

[54] T. Stöferle, H. Moritz, C. Schori, M. Köhl and T. Esslinger, Transition from a Strongly Interacting 1D Superfluid to a Mott Insulator, Phys. Rev. Lett. 92 (2004) 130403 [cond-mat/0312440] [INSPIRE].

[55] G. Birkl, M. Gatzke, I.H. Deutsch, S.L. Rolston and W.D. Phillips, Bragg scattering from atoms in optical lattices, Phys. Rev. Lett. 75 (1995) 2823.

[56] M. Weidemüller, A. Hemmerich, A. Görlitz, T. Esslinger and T.W. Hänsch, Bragg diffraction in an atomic lattice bound by light, Phys. Rev. Lett. 75 (1995) 4583.

[57] J. Ruostekoski, C.J. Foot and A.B. Deb, Light scattering for thermometry of fermionic atoms in an optical lattice, Phys. Rev. Lett. 103 (2009) 170404.

[58] S.-C. Ji et al., Softening of roton and phonon modes in a bose-einstein condensate with spin-orbit coupling, Phys. Rev. Lett. 114 (2015) 105301.

[59] J. Ye, J.M. Zhang, W.M. Liu, K. Zhang, Y. Li and W. Zhang, Light-scattering detection of quantum phases of ultracold atoms in optical lattices, Phys. Rev. A 83 (2011) 051604 [arXiv: 1001.3230] [INSPIRE].

[60] J. Ye, K.Y. Zhang, Y. Li, Y. Chen and W.P. Zhang, Optical Bragg, atomic Bragg and cavity QED detections of quantum phases and excitation spectra of ultracold atoms in bipartite and frustrated optical lattices, Annals Phys. 328 (2013) 103 [arXiv:0812.4077] [INSPIRE].

[61] M. Endres et al., The 'Higgs' Amplitude Mode at the Two-Dimensional Superfluid-Mott Insulator Transition, Nature 487 (2012) 454 [arXiv: 1204.5183] [INSPIRE].

[62] J. Léonard, A. Morales, P. Zupancic, T. Donner and T. Esslinger, Monitoring and manipulating Higgs and Goldstone modes in a supersolid quantum gas, Science 358 (2017) 1415.

[63] R.D. Peccei, The Strong CP problem and axions, Lect. Notes Phys. 741 (2008) 3 [hep-ph/0607268] [INSPIRE].

[64] R.D. Peccei and H.R. Quinn, CP conservation in the presence of pseudoparticles, Phys. Rev. Lett. 38 (1977) 1440 [INSPIRE].

[65] R.D. Peccei and H.R. Quinn, Constraints Imposed by CP Conservation in the Presence of Instantons, Phys. Rev. D 16 (1977) 1791 [InSPIRE].

[66] Y. Chen and J. Ye, Characterizing symmetry breaking patterns in a lattice by dual degrees of freedom, Phil. Mag. 92 (2012) 4484.

[67] J. Ye and Y. Chen, Quantum phases, supersolids and quantum phase transitions of interacting bosons in frustrated lattices, Nucl. Phys. B 869 (2013) 242 [cond-mat/0612009] [INSPIRE]. 